\title{
Rapid MALDI-TOF-MS Detection of Anthocyanins in Wine and Grape Using Different Matrices
}

\author{
Violeta Ivanova • Ágnes Dörnyei • Marina Stefova • \\ Trajće Stafilov • Borimir Vojnoski • Ferenc Kilár • \\ László Márk
}

Received: 16 March 2010 / Accepted: 6 April 2010 /Published online: 22 April 2010

(C) Springer Science+Business Media, LLC 2010

\begin{abstract}
Matrix-assisted laser desorption/ionization timeof-flight mass spectrometry (MALDI-TOF-MS) as a new valuable technique has been used for qualitative screening of anthocyanins in wine and grape samples from Vranec and Merlot variety. For that purpose, different MALDI matrices have been tested, such as $\alpha$-cyano-4-hydroxycinnamic acid, sinapic acid, 2,5-dihydroxybenzoic acid (2,5$\mathrm{DHB}$ ), and $\mathrm{C}_{70}$ fullerene. It was observed that 2,5-DHB was superior with respect to all the matrices used in the study. Furthermore, fullerene was, for the first time, tested
\end{abstract}

V. Ivanova $\cdot$ M. Stefova $\cdot$ T. Stafilov

Institute of Chemistry, Faculty of Natural Sciences

and Mathematics, Ss Cyril and Methodius University,

Skopje, Arhimedova 5,

Republic of Macedonia

V. Ivanova $(\bowtie) \cdot$ B. Vojnoski

Department for Enology, Institute of Agriculture,

Ss Cyril and Methodius University,

Skopje, Aleksandar Makedonski bb,

Republic of Macedonia

e-mail: violeta.ivanova@zeminst.edu.mk

Á. Dörnyei · F. Kilár

Department of Analytical and Environmental Chemistry,

Faculty of Sciences, University of Pécs,

Ifjúság útja 6 ,

7633 Pécs, Hungary

F. Kilár

Institute of Bioanalysis, Faculty of Medicine, University of Pécs,

Szigeti út 12 ,

7624 Pécs, Hungary

\section{Márk}

Department of Biochemistry and Medical Chemistry,

Faculty of Medicine, University of Pécs,

Szigeti út 12,

7624 Pécs, Hungary and used as a possible matrix for MALDI-TOF-MS identification of anthocyanins in wine and grape samples. The results obtained showed identification of aglycone forms of anthocyanins only, since the higher laser energy applied for ionization of the fullerene molecules led to great deal of fragmentation of the sugar moiety from glucosides, acetylglucoside, and $p$-coumaroylglucoside groups from the corresponding anthocyanin molecules. Additionally, liquid chromatography/electrospray ionization mass spectrometry has also been applied for anthocyanin characterization.

Keywords Anthocyanins $\cdot 2,5$-DHB $\cdot \mathrm{CHCA} \cdot \mathrm{SA} \cdot$ Fullerene $\cdot$ MALDI-TOF-MS $\cdot$ LC-MS $\cdot$ Wine $\cdot$ Grape

\section{Introduction}

Phenolic compounds are considered as main factors responsible for the quality of grapes, and thus for their corresponding wines. Among them, the anthocyanin profiles are highly variable and typical of the grape variety; therefore, they can be used as a criterion for the assessment of grape authenticity, including varietal characterization and differentiation of red grapes. Anthocyanins from Vitis vinifera grape are characterized as 3-monoglycosides, 3-acetylglycosides, 3-p-coumaroylglycosides, and 3-caffeoylglycosides of cyanidin, delphinidin, peonidin, petunidin, and the dominant malvidin (Wulf and Nagel 1978). The ratio of acetylated/p-coumaroylated anthocyanins and the sum of acylated anthocyanins have proven to be particularly significant and characteristic for certain grape varieties (Bakker and Timberlake 1985). Since the quality of grape and wine strongly depends on the anthocyanin content, obtaining information on chemical structures accomplished by molecular mass determination can provide a solid basis in order to distinguish $V$. vinifera 
species against grapevines from other species or hybrid cross-breeds.

Reversed-phase liquid chromatography (RP-HPLC) coupled with photodiode array detector is the most commonly used technique for analysis of anthocyanins (Wulf and Nagel 1978; Piovan et al. 1998; De Villers et al. 2004; Alcalde-Eon et al. 2006; Kelebek et al. 2007). Application of modern mass spectrometry techniques, such as liquid chromatography mass spectrometry (LC-MS) and matrixassisted laser desorption/ionization time-of-flight mass spectrometry (MALDI-TOF-MS), became the most sophisticated techniques for anthocyanin analyses in grape and wine. Mass spectrometry technique is applicable and effective for studies of glycoside compounds, allowing characterization of the aglycone and sugar moiety (PérezMagariño et al. 1999, de Villers et al. 2004, Monagas et al. 2005; Baldi et al. 1995, Cameira-dos-Santos et al. 1996, Wang and Sporns 1999, Revilla and González-San José 2001, Núñez et al. 2004).

Application of MALDI-TOF for analyses of anthocyanins in food samples has already been proved (Wang and Sporns 1999, Krueger et al. 2000; Carpentieri et al. 2007; Es-Safi et al. 2006; Reed et al. 2005; Robards 2003; Sugui et al. 1998; Sugui et al. 1999; Tholey and Heinzle 2006; Lerouxel et al. 2002; Yamada et al. 2007; Fraser et al. 2007). This technique allows successful determination of the molecular weights in complex samples and amount of each species, directly from ion abundances in the mass spectrum without previous isolation or clean-up of the sample (Hanton 2001). MALDI technique, for the first time was demonstrated by Karas et al. 1987, originally developed for large biomolecules analysis. Coupling of MALDI with one of the simplest mass analyzers, time-of-flight, allowed sensitive and efficient technique with high sensitivity, ease of used, speed of analysis, good tolerance towards contaminants, and, the most important, the ability to analyze complex mixtures, giving access to a theoretically unlimited mass range. A basic characteristic of MALDI is mixing the analyte with a suitable matrix, followed by application of the mixture on a MALDI plate, and then, irradiation by a pulsed laser. During the irradiation, the analyte and matrix molecules are ablated and ionized. A number of substances have been tested and applied as MALDI matrices, but there are no general rules for predicting the suitability of a substance as matrix. The most frequently used matrices are derivatives of benzoic acid, e.g., 2,5-dihydroxybenzoic acid (2,5-DHB; Juhasz et al. 1993; Carpentieri et al. 2007) and derivatives of cinnamic acid, e.g., $\alpha$-cyano-4-hydroxycinnamic acid (CHCA) or sinapic acid (SA). Fullerenes have also been used as matrices, whereas the first application was for laser desorption of biomolecules involving application of protein analyte solution directly onto the pre-deposited fullerene film (Michalak et al. 1994). Fullerene matrices has been used for MALDI-TOF analysis of small hydrophobic molecules (e.g., steroids, fatty acids; Mernyak et al. 2008, Mernyak et al. 2009), but until now there are no data reported on their application for analyses of anthocyanins (which contain hydrophobic aromatic rings) in wine and grape samples.

The most widely used sample preparation methods for MALDI analysis are the dried droplet technique when a mixed solution of sample or analyte/s and matrix is deposited onto a MALDI plate, allowed to dry, and the sandwich method where the sample is placed "in a sandwich" between two matrix layers. In this study, both preparation MALDI methods have been used in order to find the best way of preparation and the most appropriate matrix for analysis of anthocyanins in grape and wine samples. For that purpose, the first step of the work was testing the efficiency of different MALDI matrices, and then, fullerene was for the first time used and tested as a possible matrix for anthocyanin analyses applying the "sandwich" method for sample crystallization. Additionally, LC-MS technique was applied for structural characterization of anthocyanins in the samples. Thus, this research work reports a simple and fast method for anthocyanin identification in grape and wine, considered as the key compounds in enology and viticulture necessary to be monitored.

\section{Materials and Methods}

\section{Chemicals}

The MALDI matrices: $\alpha$-cyano-4-hydroxycinnamic acid, 2,5-dihydroxybenzoic acid, sinapic acid, and $\mathrm{C}_{70}$ fullerene (Gold grade) were purchased from Hoechst AG (Frankfurt, Germany). The standard of malvidin-3-glucoside was purchased from LGC Promochem GmbH, Szentendre (Hungary). Methanol and acetonitrile (HPLC grade) were purchased from Scharlau Chemie S.A. All the other used reagents were of analytical purity grade.

\section{Grape Samples}

Grape berries used for this study were grown at the vineyards of the Institute of Agriculture in Skopje. Samples from Vranec variety (harvest 2007) were collected from 8 years old vineyards (13.4 ha) when the grapes were at technological ripeness, performing random sampling (10 kg from 30-40 plants) by picking berries from the top, central, and bottom parts of the cluster. Samples were kept on $-20^{\circ}$ $\mathrm{C}$ before analysis. 


\section{Extraction Procedure}

Stems were removed and while the grapes were still frozen, skins were separated from the pulps. The skins, thawed off, were blotted on paper towels to remove any residual pulp and were grounded. One gram of skins were extracted twice in closed Erlenmeyers $(25 \mathrm{~mL})$ with $10 \mathrm{~mL}$ acetone/water $(80 / 20, V / V)$ containing $\mathrm{HCl}(0.1 / 10, V / V)$ to prevent oxidation of polyphenols (Ivanova et al. 2010). Extraction started with maintaining for $15 \mathrm{~min}$ in an ultrasonic bath and then stirred for $30 \mathrm{~min}$ on a magnetic stirrer. The mixture was centrifuged at 3,000 rpm for $10 \mathrm{~min}$. For each extraction, the supernatants were collected, joined, and brought to a final volume of $25 \mathrm{~mL}$ with distilled $\mathrm{H}_{2} \mathrm{O}$ and then, concentrated by rotoevaporation.

\section{Wine Samples}

Vranec and Merlot wines (vintage 2007) kindly provided by the Department for Enology, Institute of Agriculture in Skopje, Republic of Macedonia, were assessed over this study.

\section{MALDI-TOF-MS Analysis}

MALDI-TOF-MS analyses were performed using the Autoflex II. MALDI-TOF/TOF-MS instrument (Bruker, Daltonics, Bremen, Germany), scanning in positive and reflectron mode in the range of $50-700 \mathrm{~m} / \mathrm{z}$ and accumulating data from 1,000 consecutive laser shots. The samples were crystallized with the different matrices (CHCA, SA, 2,5-DHB, and $\mathrm{C}_{70}$ fullerene) and were ionized by nitrogen laser pulse $(\lambda=337 \mathrm{~nm}, 50 \mathrm{~Hz})$ accelerated under $20 \mathrm{kV}$. The laser power was adjusted to $\sim 60 \%$ of its maximal intensity in case of fullerene matrix, otherwise it was between $30 \%$ and $45 \%$.

Ten milligrams of the matrices were dissolved in $1 \mathrm{~mL}$ $0.1 \% \mathrm{TFA} / \mathrm{ACN}(2: 1, V / V)$ and the saturated solution of fullerene was prepared in toluene. One microliter of sample solutions were loaded onto the target plate (MTP 384 massive target plate, Bruker Daltonics, Bremen, Germany) by mixing with the same volume $(1 \mu \mathrm{L})$ of the matrix solutions. Sandwich method was used with $\mathrm{C}_{70}$ fullerene matrix. One microliter of the fullerene solution was loaded onto the target plate and then the sample droplet $(1 \mu \mathrm{L})$ was spotted on top of the evaporated matrix and finally it was covered by the second matrix layer $(1 \mu \mathrm{L})$. Internal calibration was carried out for the samples mixed with CHCA, SA, and 2,5-DHB by using the monoisotopic peaks of the positively charged quasimolecular and dimer ions of matrices. External calibration was performed with standard solution of the mixture of CHCA and SA in case of using fullerene as a matrix. Data processing was executed with
FlexAnalysis 2.4 software packages. The components were identified according to the molecular mass of their monoisotopic molecular or quasimolecular ions.

\section{HPLC Analysis}

For identification purposes, an Agilent Series 1100 LC System combined with an Agilent 6300 Series Ion Trap LC-MS system was used, equipped with a binary pump, a degasser, an autosampler (100 $\mu \mathrm{L}$ sample loop), a column thermostat, and UV-Vis diode-array detector for identification of phenolic compounds. Separation of the components was performed on a Phenomenex RP C18 column (60× $4.6 \mathrm{~mm}$, i.d. $3 \mu \mathrm{m}$ ) with a mobile phase consisting of $1 \%$ $(V / V)$ acetic acid in water (solvent $\mathrm{A})$ and $1 \%(V / V)$ acetic acid in methanol (solvent $\mathrm{B}$ ) at flow rate of $0.2 \mathrm{~mL} / \mathrm{min}$ and at $25^{\circ} \mathrm{C}$. Proportions of solvent $\mathrm{B}$ were as follows: 0-10 min, 5-20\%; 10-45 min, 20-50\%; 45-50 min, 50$80 \%$; and $50-60 \mathrm{~min}, 80-90 \%$. The HPLC system was connected to the mass spectrometer equipped with an electrospray ion source, operated in alternating (positive and negative) mode. Nitrogen was used as drying gas at $325^{\circ} \mathrm{C}$, with a flow rate of $5 \mathrm{~L} / \mathrm{min}$; the pressure of the nebulizer was set at $15 \mathrm{psi}$. The scanning mass to charge range of the ion trap was $50-2,200 \mathrm{~m} / \mathrm{z}$ with a maximum accumulation time of $200 \mathrm{~ms}$.

\section{Results and Discussion}

The primary aim of this study was to apply a MALDI-TOFMS method for fast throughput analysis of wine and grape anthocyanins. Since the choice of the matrix and the sample preparation are two critical concerns for successful MALDI analysis, different MALDI matrices have been tested and their effects compared in order to check their suitability for identification of anthocyanins. Thus, three matrices, including CHCA, SA, and 2,5-DHB, prepared with the dried droplet technique, and $\mathrm{C}_{70}$ fullerene prepared with the sandwich method, have been tested. Their positive-ion MALDI-TOF-MS spectra are shown in Fig. 1.

As can be seen from the figure, comparing the TOF-MS spectra of the different matrices used, it was observed that the obtained quasimolecular, fragment, and adduct ion peaks for CHCA, SA, and 2,5-DHB appeared in the mass range of $\mathrm{m} / \mathrm{z} 100-600$ showing almost the same intensity. Opposite, the peaks of $\mathrm{C}_{70}$ fullerene matrix practically did not show peaks in this range, which was expected since the molecular mass of fullerene is $m / z 840$, meaning that even a few obtained signal peaks would have very low intensity in the range of interest for anthocyanin identification $(\mathrm{m} / \mathrm{z}$ 100-700), as it was observed. Moreover, giving good quality spectra, appeared fullerene matrix peaks with very 

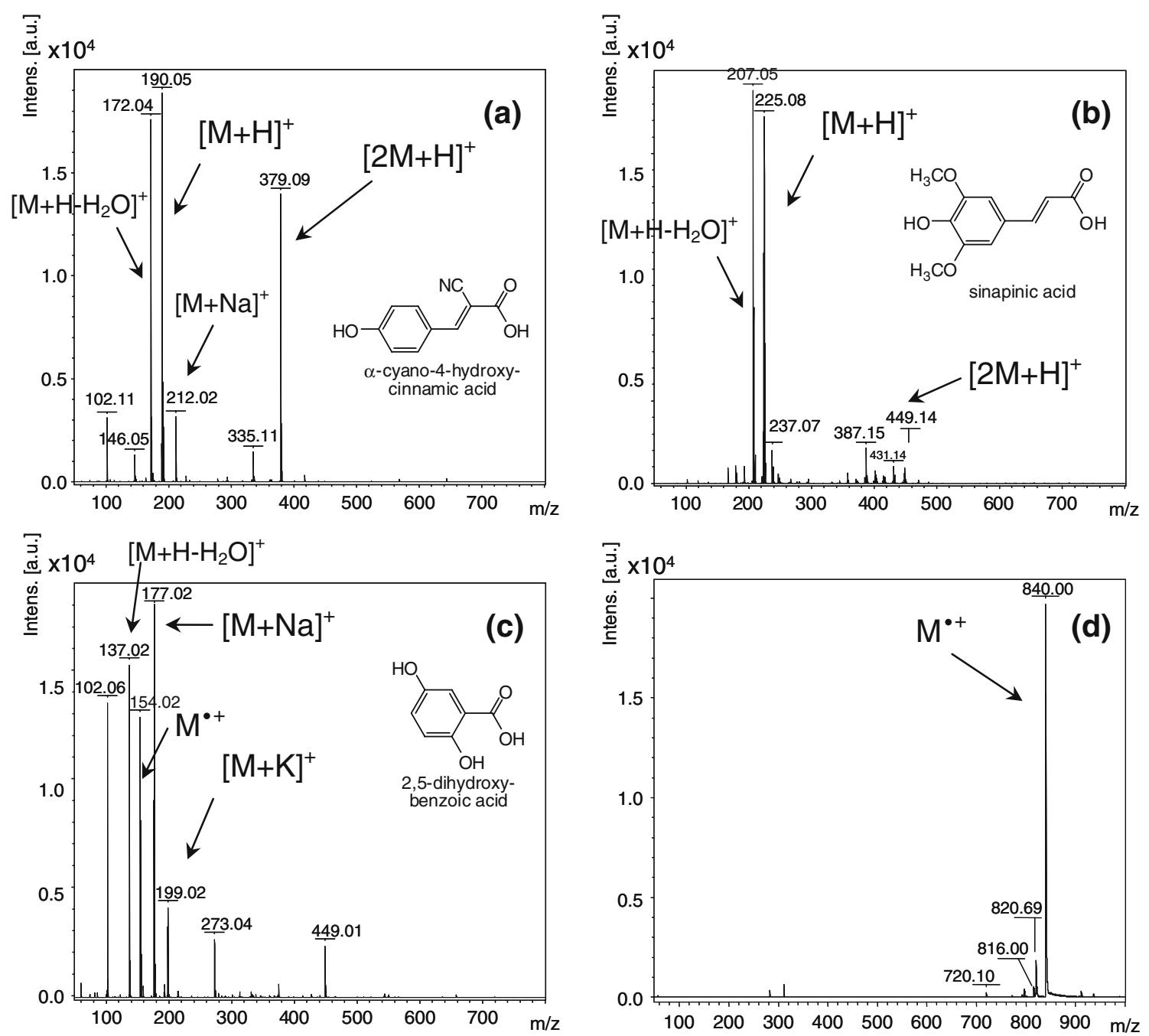

Fig. 1 Positive-ion MALDI-TOF mass spectra of the matrices. a CHCA ( $\alpha$-cyano-4-hydroxycinnamic acid), b SA (sinapic acid), c 2,5-DHB (2,5-dihydroxybenzoic acid), and $\mathbf{d} \mathrm{C}_{70}$ fullerene

low intensity in this low range $(\mathrm{m} / \mathrm{z} 100-700)$ could not influence the identification of the sample anthocyanin peaks and interfere with them. Therefore, fullerene was tested as a possible new matrix for wine and grape anthocyanin analyses, together with the other three well-known MALDI matrices. The obtained MALDI-TOF-MS results, presented as relative intensities (\%) of the anthocyanin peaks, detected in Vranec grape skin extract and two wines, Vranec and Merlot, analyzed with the different matrices, are presented in Table 1. MALDI-TOF-MS spectra of Vranec skin extract obtained with different MALDI matrices tested are presented in Fig. 2.

As can be seen from Table 1 and Fig. 2, it was observed that 2,5-DHB was superior for identification of anthocyanins present in the analyzed samples, with respect to all the matrices tested. In fact, DHB prepared in $0.1 \%$ TFA/ACN $(2: 1, V / V)$ allowed homogeneous sample preparation and higher ionization efficiency. The big sensitivity of the MALDI technique, allowed identification of the anthocyanins in their glucoside, acetylglucoside, and $p$-coumaroylglucoside forms present in the grape skin and wine, as well as identification of the five aglycones. Results were in agreement with the previously published results (Carpentieri et al. 2007) whereas DHB showed best results with respect to all the matrices tested in the study. Figure 3 shows the MALDI-TOF-MS spectra of skin extract and two wine samples obtained with 2,5-DHB matrix.

Fullerene matrix was, for the first time, used for anthocyanin analyses applying the sandwich method, whereas the analyte is basically sandwiched between two matrix layers, which gave the possibility to analyze hydroscopic samples. MALDI-TOF-MS "fingerprints" of the analyzed wine and grape samples, applying sandwich method with $\mathrm{C}_{70}$ fullerene matrix for ionization, are shown in Fig. 4. 
Table 1 Relative intensities (\%) of the anthocyanin peaks identified by MALDI-TOF-MS in grape and wine samples applying different matrices

\begin{tabular}{|c|c|c|c|c|c|c|c|c|c|c|c|c|c|c|c|c|c|c|c|c|}
\hline \multirow{2}{*}{$\begin{array}{l}\text { Anthocyanins } \\
\text { (rel. intens. \%) } \\
\text { Matrix }\end{array}$} & $\mathrm{Dp}$ & $\mathrm{Cy}$ & $\mathrm{Pt}$ & $\mathrm{Pn}$ & $\mathrm{Mv}$ & $\mathrm{Dp}$ & $\mathrm{Cy}$ & $\mathrm{Pt}$ & Pn & $\mathrm{Mv}$ & $\mathrm{Dp}$ & $\mathrm{Cy}$ & $\mathrm{Pt}$ & Pn & $\mathrm{Mv}$ & $\mathrm{Dp}$ & $\mathrm{Cy}$ & $\mathrm{Pt}$ & Pn & $\mathrm{Mv}$ \\
\hline & \multicolumn{5}{|c|}{ Aglycones } & \multicolumn{5}{|c|}{ Glucosides } & \multicolumn{5}{|c|}{ Acetylglucosides } & \multicolumn{5}{|c|}{$p$-coumaroylglucosides } \\
\hline \multicolumn{21}{|c|}{ Grape skin extract } \\
\hline CHCA & $<1$ & $<1$ & 5 & 4 & 100 & - & - & - & - & 2 & - & - & - & - & $<1$ & - & - & $<1$ & $<1$ & 1 \\
\hline SA & $<1$ & - & $<1$ & $<1$ & 10 & - & 1 & $<1$ & $<1$ & 11 & - & - & - & - & 2 & - & - & 1 & 1 & 27 \\
\hline 2,5-DHB & $<1$ & $<1$ & 3 & 3 & 41 & - & - & 2 & 3 & 78 & - & - & $<1$ & $<1$ & 19 & - & - & 5 & 4 & 83 \\
\hline $\mathrm{C}_{70}$ sandwich & 3 & 2 & 9 & 8 & 90 & - & - & - & - & - & - & - & - & - & - & - & - & - & - & - \\
\hline \multicolumn{21}{|l|}{ Vranec wine } \\
\hline CHCA & 1 & $<1$ & 4 & 5 & 72 & - & - & - & $<1$ & 9 & - & - & - & - & 1 & - & - & - & - & $<1$ \\
\hline SA & $<1$ & - & $<1$ & $<1$ & 8 & - & - & - & - & 19 & - & - & - & - & 3 & - & - & $<1$ & $<1$ & 14 \\
\hline 2,5-DHB & 1 & - & 1 & 1 & 14 & - & - & 1 & 2 & 30 & - & - & - & $<1$ & 6 & - & $<1$ & $<1$ & 1 & 10 \\
\hline $\mathrm{C}_{70}$ sandwich & $<1$ & $<1$ & 1 & 2 & 35 & - & - & - & - & - & - & - & - & - & - & - & - & - & - & - \\
\hline \multicolumn{21}{|l|}{ Merlot wine } \\
\hline $\mathrm{CHCA}$ & $<1$ & - & 1 & 2 & 43 & - & - & - & $<1$ & 4 & - & - & - & - & $<1$ & - & - & - & - & $<1$ \\
\hline SA & 1 & - & 1 & 1 & 25 & - & - & $<1$ & $<1$ & 20 & - & - & - & - & 4 & - & - & $<1$ & $<1$ & 18 \\
\hline 2,5-DHB & 1 & - & $<1$ & $<1$ & 5 & $<1$ & - & $<1$ & 1 & 13 & - & - & - & $<1$ & 2 & - & - & $<1$ & $<1$ & 4 \\
\hline $\mathrm{C}_{70}$ sandwich & 1 & $<1$ & 2 & 5 & 100 & - & - & - & - & - & - & - & - & - & - & - & - & - & - & - \\
\hline
\end{tabular}

CHCA $\alpha$-cyano-4-hydroxycinnamic acid, $S A$ sinapic acid, 2,5-DHB 2,5-dihydroxybenzoic acid, $C_{70}$ fullerene

Applying this matrix for ionization, anthocyanins were identified in their agylcone forms (Figs. 2 and 4). Actually, the strength of the laser energy was tested and observed that ionization of the matrix molecules required higher energy in order to perform fragmentation of the matrix molecules. Nevertheless, adjusting the laser power to $\sim 60 \%$ of its maximal intensity caused fragmentation of the sample molecules, leading great deal of fragmentation of the sugar moiety from glucosides, acetylglucoside, and $p$-coumaroylglucoside groups from the corresponding anthocyanin molecules resulting with elimination of the glucoside, acetylglucoside, and p-coumaroylglucoside groups, thus allowing identification of the anthocyanins only in their aglycon forms. Taking into account the results from this research, we could conclude that the attempt of using fullerene as a possible matrix for MALDI screening of anthocyanins should be extended on testing the effect of derivatized and/or acidified fullerenes as possible matrices, since those matrices require lower laser power for ionization. Furthermore, the fullerene derivatives, as a new class of matrices, possesses high analyte ionization efficiency, small molar ratios (less than 1) of matrix/analyte and a broader optical absorption spectrum, which should obviate specific wavelength lasers for MALDI acquisitions (Ugarov et al. 2004) and could be used for MALDI screening of anthocyanins. Thus, further investigations are necessary to be performed in order to allow proper identification of grape and wine anthocyanins (glycosides, acetyl, and $p$ coumaroyl derivatives) applying the new MALDI matrices.
Identification of the peaks from the analyzed samples, obtained with application of the different MALDI matrices was performed on the basis of the targeted fragmentation of the ions of interest (their $\mathrm{M}^{+}$signals) under positive-ion mode. Thus, positively charged flavylium cation of malvidin $(m / z$ 331) was detected in all samples under each experimental condition. Other anthocyanin aglycones, including delphinidin, cyanidin, petunidin, and peonidin, have been detected in the wine and grape skin samples, observing fragment ions of $\mathrm{M}^{+}$at $m / z 303,287,317$, and 301 , respectively. The molecular ion peaks that appeared in the mass spectrum, at $\mathrm{m} / \mathrm{z} 493,535$, and 639 corresponded to the malvidin-3-glucoside, malvidin-3-acetylglucoside, and malvidin-3- $p$-coumaroylglucoside, respectively. Molecular peaks at $\mathrm{m} / \mathrm{z} 317,479$, and 625 exhibited in the MALDI-TOF-MS spectrum of the grape skin extract, obtained with 2,5-DHB matrix and have been identified as petunidin, petunidin-3-glucoside, and petunidin-3-p-coumaroylglucoside, respectively. Compounds peonidin, peonidin-3-glucoside, and peonidin-3-p-coumaroylglucoside have been detected on the basis of their $\mathrm{M}^{+}$signals at $\mathrm{m} / \mathrm{z} 301,463$, and 609 , respectively.

In order to confirm the obtained MALDI-MS results for the presence of glucoside, acetylglucoside, and $p$-coumaroylglucoside derivatives of delphinidin, cyanidin, petunidin, peonidin, and malvidin in the skin extract and wines, additional LC-MS analysis has been performed. UV-Vis spectra, molecular and fragment ions of delphinidin-3glucoside, cyanidin-3-glucoside, petunidin-3-glucoside, 

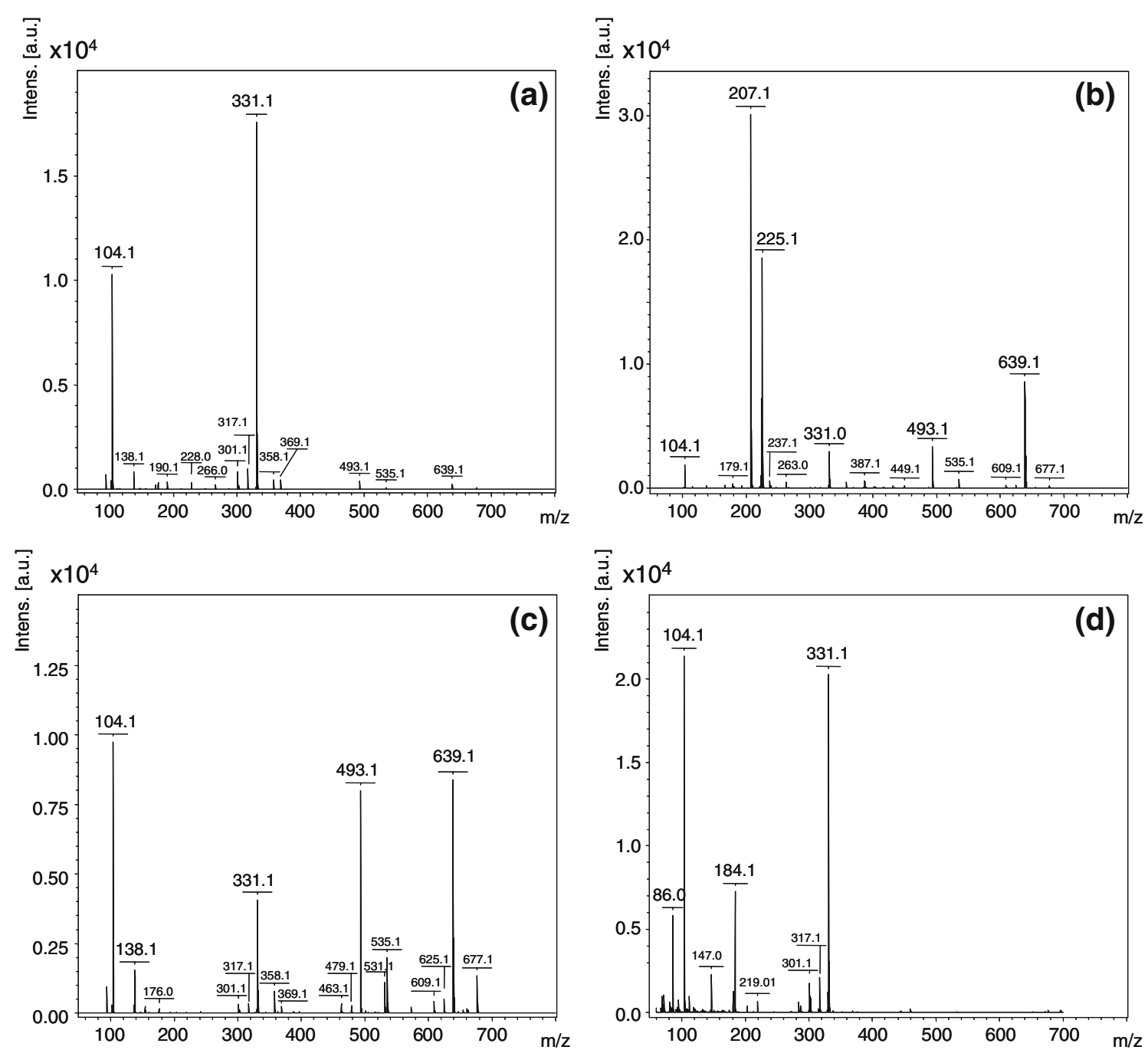

Fig. 2 Positive-ion MALDI-TOF mass spectra of the grape skin extract of Vranec variety obtained with different MALDI matrices: a CHCA ( $\alpha$ cyano-4-hydroxycinnamic acid), b SA (sinapic acid), c 2,5-DHB (2,5-dihydroxybenzoic acid), and d C 70 fullerene ("sandwich" method)

peonidin-3-glucoside, and the dominant malvidin-3glucoside, were in agreement with the literature data (Vivar-Quintana et al. 2002; Alcalde-Eon et al. 2004, De Villers et al. 2004, Wu and Prior 2005; Chinnici et al. Chinnici et al. 2009). Molecular and fragmentation ions, as well as $\lambda_{\max }$ values are presented in Table 2. The monoglucosides have similar mass spectra, characterized with two signals, molecular ion $\mathrm{M}^{+}$, and aglycone fragment $[\mathrm{M}-162]^{+}$which results from elimination of glucose molecule. The anthocyanin derivatives, 3-acetylglucosides and 3-p-coumaroylglucosides, of the five anthocyanins were identified in a similar way. The mass spectra of 3acetylglucosides are characterized with $\mathrm{M}^{+}$and $[\mathrm{M}-204]^{+}$ fragment. The fragment ion at $204 \mathrm{Da}$ corresponds to the acetylglucoside group, so the fragment $[\mathrm{M}-204]^{+}$corre- sponds to the related aglycone and $p$-coumaroylglucoside derivatives give two signals, the molecular ion $\mathrm{M}^{+}$and fragment ion $[\mathrm{M}-308]^{+}$, as a result of loss of $p$-coumaroylglucoside group.

\section{Conclusions}

Rapid and simple MALDI-TOF-MS method, without sample preparation, was used for identification of anthocyanins in grape and wine, demonstrating the ability of MALDI as valuable technique for fast screening. Different matrices, including $\mathrm{CHCA}, \mathrm{SA}, 2,5-\mathrm{DHB}$, and $\mathrm{C}_{70}$ fullerene have been tested, observing best results with 


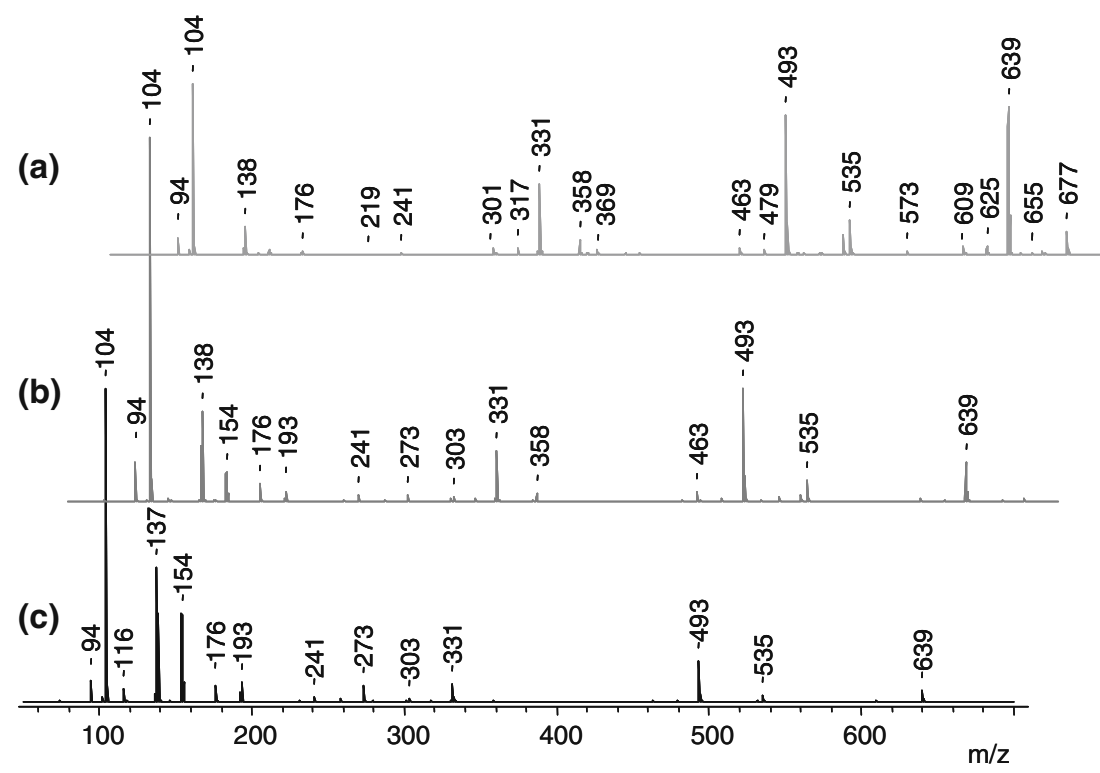

Fig. 3 Positive-ion MALDI-TOF mass spectra of: a Vranec grape skin extract, b Vranec wine, and c Merlot wine, obtained in presence of 2,5DHB matrix

2,5-DHB matrix for ionization, that allowed identification and confirmation of the presence of the dominant compounds in wine and skin samples, such as malvidin and its derivatives: malvidin-3-glucoside, malvidin-3acetylglucoside, and malvidin-3-p-coumaroylglucoside, considered as the most important compounds for the quality and color of grapes and wines. Skin extracts and wine samples were analyzed without additional purification confirming the ability of MALDI-TOF-MS to analyze

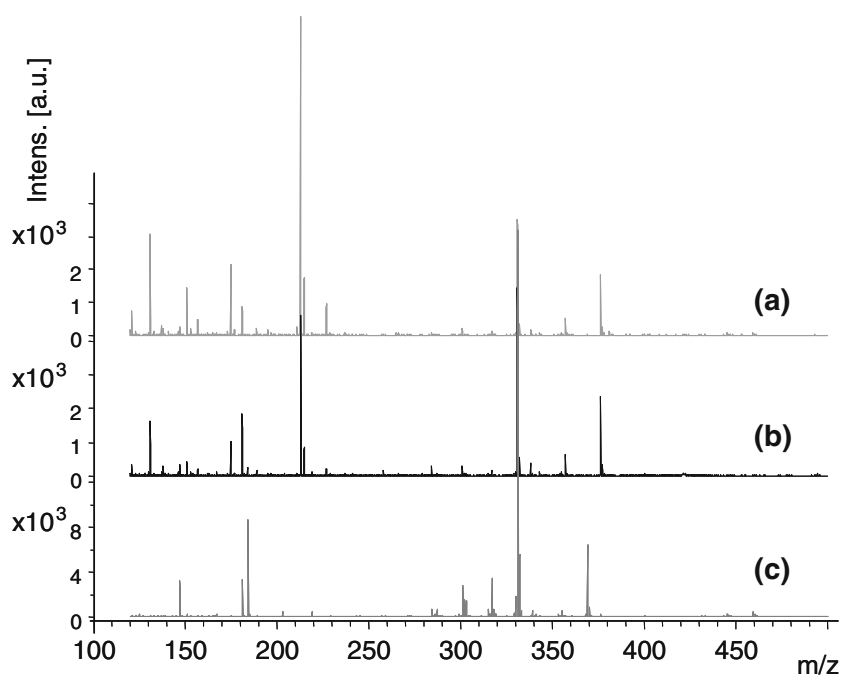

Fig. 4 Positive-ion MALDI-TOF mass "fingerprint" spectra of the analyzed samples: a Vranec wine, b Merlot wine, c skin extract of Vranec grape obtained with $\mathrm{C}_{70}$ fullerene "sandwich" matrix crude samples. Fullerene was for the first time applied as a possible matrix for MALDI-TOF-MS analysis of anthocyanins in grape and wines, but, great deal of fragmentation of sugar moiety was observed, concluding that further extended investigations should be performed on testing derivatized fullerenes, as a possible new matrices suitable for MALDI-TOF-MS analysis of anthocyanins.

Table 2 Anthocyanins identified with LC-MS

\begin{tabular}{llccc}
\hline$t_{\mathrm{R}}$ & Anthocyanins & $\lambda_{\text {max }} / \mathrm{nm}$ & $M^{+}$ & Fragments $(\mathrm{m} / \mathrm{z})$ \\
\hline 21.4 & Dp-3-glc & 525 & 465 & 303 \\
23.7 & Cy-3-glc & 517 & 449 & 287 \\
25.2 & Pt-3-glc & 525 & 479 & 317 \\
27.6 & Pn-3-glc & 516 & 463 & 301 \\
28.4 & Mv-3-glc & 528 & 493 & 331 \\
33.6 & Dp-3-acetylglc & 530 & 507 & 303 \\
36.2 & Cy-3-acetylglc & 532 & 491 & 287 \\
36.9 & Pt-3-acetylglc & 535 & 521 & 317 \\
39.7 & Pn-3-acetylglc & 525 & 505 & 301 \\
39.5 & Mv-3-acetylglc & 528 & 535 & 331 \\
43.9 & Dp-3- $p$-coumglc & 530 & 611 & 303 \\
46.3 & Cy-3-p-coumglc & & 595 & 287 \\
46.8 & Pt-3- $p$-coumglc & 528 & 625 & 317 \\
49.0 & Pn-3- $p$-coumglc & 523 & 609 & 301 \\
49.2 & Mv-3- $p$-coumglc & 530 & 639 & 331 \\
\hline
\end{tabular}

$D p$ delphinidin, $C y$ cyanidin, $P t$ petunidin, $P n$ peonidin, $M v$ malvidin, glc glucose, aclglc acetylglucoside, $p$-coumglc p-coumaroylglucoside 
Acknowledgments The authors acknowledge the CEEPUS Program realized through the CEEPUS network (CIIHU-0010-01-0607-Teaching and learning bioanalysis) for the given opportunity and financial support for performing the MALDI-TOF-MS analyses at Department of Biochemistry and Medical Chemistry, Faculty of Medicine, at the University of Pécs, Hungary. The work was also supported by the grants GVOP-3.2.1-0168, GVOP-3.2.1-0179, RET 008/2005, OTKA 46476, OTKA-NKTH NI-68863, and DDEK Nonprofit Kft.

\section{References}

Wulf LW, Nagel CW (1978) Am J Enol Viticult 29:42

Bakker J, Timberlake CF (1985) Am J Enol Viticult 36(3):252-253

Piovan A, Filippini R, Favretto D (1998) Rapid Commun Mass Spectrom 12:361

de Villers A, Vanhoenacker G, Majek P, Sandra P (2004) J Chromatogr A 1054:195

Alcalde-Eon C, Escribano-Bailón MT, Santos-Buelga C, RivasGonzalo JC (2006) Anal Chim Acta 563:238

Kelebek H, Canbas A, Selli S (2007) Chromatographia 66:207

Pérez-Magariño S, Revilla I, González-San José ML, Beltrán S (1999) J Chromatogr A 847:75

Monagas M, Suárez R, Gómez-Cordovés C, Bartolomé B (2005) Am J Enol Viticult 56:139

La Torre GL, Saitta M, Vilasi F, Pellicanò T, Dugo G (2006) Food Chem 94:640

Baldi A, Romani A, Mulinacci N, Vincieri FF, Casetta B (1995) J Agric Food Chem 43:2104

Cameira-dos-Santos PJ, Brillouet JM, Cheynier V, Moutounet M (1996) J Sci Food Agric 70:204

Wang J, Sporns P (1999) J Agric Food Chem 47:2009

Revilla I, González-San José ML (2001) J Wine Res 12:183

Núñez V, Monagas M, Gómez-Cordovés C, Bartolom B (2004) Postharvest Biol Technol 31:69

Krueger CG, Dopke NC, Treichel PM, Folts J, Reed JD (2000) J Agric Food Chem 48:1663
Carpentieri A, Marino G, Amoresano A (2007) Anal Bioanal Chem 389:969

Es-Safi NE, Guyot S, Ducrot PH (2006) J Agric Food Chem 54:6969

Reed JD, Krueger CG, Vestling MM (2005) Phytochem 66:2248

Robards K (2003) J Chromatogr A 1000:657

Sugui JA, Bonham C, Lo SC, Wood KV, Nicholson RL (1998) Phytochem 8:1063

Sugui JA, Wood KV, Yang ZY, Bonham CC, Nicholson RL (1999) Am J Enol Viticult 50:199

Tholey A, Heinzle E (2006) Anal Bioanal Chem 386:24

Lerouxel O, Choo TS, Séveno M, Usadel B, Faye L, Lerouge P, Pauly M (2002) Rapid Plant Physiol 130:1754

Yamada K, Abe T, Tanizawa Y (2007) Food Chem 103:8

Fraser PD, Enfissi EMA, Goodfellow M, Eguchi T, Bramley PM (2007) Plant J 49:552

Hanton SD (2001) Chem Rev 101:521

Karas M, Bachmann D, Bahr U, Hillenkamp F (1987) Int J Mass Spectrom Ion Processes 78:53

Juhasz P, Costello CE, Biemann K (1993) J Am Soc Mass Spectrom 4 (5):399

Michalak L, Fisher K, Alderdice D, Jardine D, Willett G (1994) Org Mass Spectrom 29:512

Mernyak E, Bikadi Z, Hazai E, Mark L, Schneider G, Wolfling J (2008) Lett Org Chem 5:17

Mernyak E, Kozma E, Hetenyi A, Mark L, Schneider G, Wolfling J (2009) Steroids 74:520

Ivanova V, Stefova M, Chinnici F (2010) J Serb Chem Soc 75:45

Ugarov MV, Egan T, Khabashesku DV, Schultz JA, Peng HQ, Khabashesku VN, Furutani H, Prather KS, Wang HWJ, Jackson SN, Woods AS (2004) Anal Chem 76(22):6734

Vivar-Quintana AM, Santos-Buelga C, Rivas-Gonzalo JC (2002) Anal Chim Acta 458:147

Alcalde-Eon C, Escribano-Bailón MT, Santos-Buelga C, RivasGonzalo JC (2004) Anal Chim Acta 513:305

Wu X, Prior RL (2005) J Agric Food Chem 53:3101

Chinnici F, Sonni F, Natali N, Galassi S, Riponi C (2009) Food Chem 113:651 